\title{
Omega-3 polyunsaturated fatty acid and ursodeoxycholic acid have an additive effect in attenuating diet-induced nonalcoholic steatohepatitis in mice
}

\author{
Ja Kyung Kim ${ }^{1}$, Kwan Sik Lee ${ }^{1}$, Dong Ki Lee ${ }^{1}$, Su Yeon Lee ${ }^{2}$, Hye Young Chang ${ }^{2}$, Junjeong Choi ${ }^{3}$
} and Jung Il Lee ${ }^{1}$

Nonalcoholic steatohepatitis (NASH) can progress into liver cirrhosis; however, no definite treatment is available. Omega-3 polyunsaturated fatty acid (omega-3) has been reported to alleviate experimental NASH, although its beneficial effect was not evident when tested clinically. Thus, this study aimed to investigate the additive effect of omega-3 and ursodeoxycholic acid (UDCA) on diet-induced NASH in mice. C57BL/6 mice were given a high-fat diet (HFD) for 24 weeks, at which point the mice were divided into three groups and fed HFD alone, HFD with omega-3 or HFD with omega-3 in combination with UDCA for another 24 weeks. Feeding mice an HFD and administering omega-3 improved histologically assessed liver fibrosis, and UDCA in combination with omega-3 further attenuated this disease. The assessment of collagen $\alpha 1$ (I) expression agreed with the histological evaluation. Omega-3 in combination with UDCA resulted in a significant attenuation of inflammation whereas administering omega-3 alone failed to improve histologically assessed liver inflammation. Quantitative analysis of tumor necrosis factor $\boldsymbol{\alpha}$ showed an additive effect of omega-3 and UDCA on liver inflammation. HFD-induced hepatic triglyceride accumulation was attenuated by omega-3 and adding UDCA accentuated this effect. In accordance with this result, the expression of sterol regulatory binding protein-1c decreased after omega-3 administration and adding UDCA further diminished SREBP-1c expression. The expression of inducible nitric oxide synthase (iNOS), which may reflect oxidative stress-induced tissue damage, was suppressed by omega-3 administration and adding UDCA further attenuated iNOS expression. These results demonstrated an additive effect of omega-3 and UDCA for alleviating fibrosis, inflammation and steatosis in diet-induced NASH.

Experimental \& Molecular Medicine (2014) 46, e127; doi:10.1038/emm.2014.90; published online 19 December 2014

\section{INTRODUCTION}

Nonalcoholic fatty liver disease, which is one of the most prevalent chronic liver diseases worldwide, consists of a spectrum of conditions that includes nonalcoholic steatohepatitis (NASH). ${ }^{1-3}$ Although simple steatosis may undergo an indolent natural course, when it is accompanied by advanced liver fibrosis it often takes a similar clinical course to that of chronic hepatitis $\mathrm{C}$ virus infection with advanced fibrosis. ${ }^{4}$ However, with all of these possible liver-associated morbidities and mortalities, a definitive treatment that would stop or reverse the progression of the disease has yet to be discovered.
Omega-3 polyunsaturated fatty acid (omega-3) is clinically used for treating hypertriglyceridemia. ${ }^{5}$ There is evidence that omega-3 suppresses hepatic triglyceride (TG) accumulation and reduces inflammation, thereby alleviating NASH in animal models. ${ }^{6-9}$ However, solid clinical evidence of the benefit of omega-3 in NASH is lacking, even though the absence of significant adverse effects of omega-3 makes it convenient to use as a supplement. ${ }^{10-12}$

Ursodeoxycholic acid (UDCA) is reported to have a beneficial role in experimental NASH because of its antiinflammatory, hepato-protective and antioxidant effects. ${ }^{13-16}$ However, clinical trials have failed to show meaningful

\footnotetext{
${ }^{1}$ Department of Internal Medicine, Yonsei University College of Medicine, Seoul, Korea; ${ }^{2}$ Medical Research Center, Gangnam Severance Hospital, Yonsei University College of Medicine, Seoul, Korea and ${ }^{3}$ Department of Pathology, Yonsei University Wonju College of Medicine, Wonju, Korea Correspondence: Professor JI Lee or Professor DK Lee, Department of Internal Medicine, Gangnam Severance Hospital, Yonsei University College of Medicine, 211 Eonju-ro Gangnam-gu, Seoul 135-720, Korea.

E-mail: mdflorence@yuhs.ac or DKLEE@yuhs.ac
}

Received 17 April 2014; revised 25 September 2014; accepted 5 October 2014 
improvement in liver inflammation or fibrosis in NASH patients treated with UDCA. ${ }^{17,18}$ Although negative results may be attributable to improperly constructed studies, including suboptimal drug dosage and study duration, it has been suggested that a combination of UDCA with other agents, such as vitamin $\mathrm{E}$, might result in clinically meaningful outcomes; ${ }^{19}$ however, a study showed that long-term consumption of high doses of vitamin E (daily dose of $400 \mathrm{IU}$ ) increased mortality. ${ }^{20}$ Safety should be a concern when combination therapy is tested.

Agents that demonstrate beneficial effects on experimental NASH often fail to show significant improvement in human studies. Therefore, a combination therapy that is safe and more effective than either agent alone might have a better outcome in clinical settings. Thus, this study aimed to investigate the effect of omega- 3 in combination with UDCA on diet-induced NASH in mice and to compare the results with those of omega-3 alone to assess the possibility of this combination as a treatment for NASH.

\section{MATERIALS AND METHODS}

\section{Ethics}

The animal experimental procedures and protocols were approved by the Institutional Animal Care and Use Committee of Gangnam Severance Hospital, Yonsei University College of Medicine (Permit Number: 0011). The study was carried out in accordance with the recommendations and restrictions of the Institutional Animal Care and Use Committee.

\section{Animals \\ Male C57BL/6 mice (12 weeks of age) were obtained from the Central Lab Animal (Seoul, Korea) and housed under a 12-h light-dark cycle. Mice were fed with a diet that consisted of $15 \%$ anhydrous milk fat, $1.0 \%$ cholesterol and 50\% sucrose (a high-fat diet (HFD)) that was purchased from Picolab (Bethlehem, PA, USA). These mice were fed with the HFD for 24 weeks, at which point they were divided into three groups and fed with HFD alone (HFD), HFD and $70 \mathrm{mg} \mathrm{kg}^{-1}$ omega-3 (Pronova Biocare, Sandefjord, Norway; HFD/Omega-3) or HFD and omega- 3 in combination with $20 \mathrm{mg} \mathrm{kg}^{-1}$ UDCA (Dae- woong Pharm., Seoul, Korea; HFD/Omega-3+UDCA) for another 24 weeks. Omega- 3 and UDCA were diluted in $0.75 \%$ Tween- 80 and administered orally through sonde for 24 weeks. After 48 weeks of HFD feeding with or without administering experimental agents, the mice were sacrificed and their livers were harvested. A sample of fresh liver tissue was fixed in 10\% buffered formalin, and the remaining tissue was snap-frozen in liquid nitrogen and stored at $-80^{\circ} \mathrm{C}$. Blood samples were collected by cardiac puncture after the mice were anesthetized and were stored at $-80^{\circ} \mathrm{C}$.}

\section{Histological evaluation}

Sections of liver tissue specimens, fixed in $10 \%$ formalin and embedded in paraffin wax, were stained with H\&E and Sirius Red for histological evaluation. A blinded pathologist evaluated the slides for inflammation as described in previous studies with minor modifications. ${ }^{21-23}$ Inflammation was histologically quantified by counting inflammatory foci in 20 consecutive high-power fields ( $\times 40$ objective; average histological grade, grade 0 : no foci, grade 1: $<2$ foci per high-power field, grade $2: \geqslant 2$ foci per high-power field). Liver fibrosis was evaluated by calculating the fibrosis ratio using an image analysis system as described in a previous study with some modifications. ${ }^{24}$ Briefly, images of Sirius Red-stained sections were captured under $\times 10$ magnification. In the stained section, red indicated fibrosis and gray indicated parenchyma. After interactive thresholding, the image was converted into a binary image. The twodimensional patterns were measured by direct pixel counting on the binary images. The total area was the sum of the areas of the microscopic fields that included parenchyma and fibrosis. For each slide, the area of fibrosis was evaluated in 20 consecutive high-power fields and was then averaged.

\section{RNA extraction and gene expression analysis by quantitative real-time PCR}

Total RNA was extracted from frozen whole liver using Trizol reagent (Invitrogen, Carlsbad, CA, USA) according to the manufacturer's protocol. RNA samples were quantified by spectrophotometry. RNA integrity was assessed using agarose gel electrophoresis and ethidium bromide staining. The RNA samples were then diluted in RNase-free water and stored at $-70{ }^{\circ} \mathrm{C}$ until use. Five micrograms of RNA was reverse transcribed using an RNA PCR kit (version 1.2; Takara Bio Inc., Japan) according to the manufacturer's recommendations. Oligonucleotide primers and TaqMan probes for collagen $\alpha 1(I)$, transforming growth factor (TGF) $\beta$, tumor necrosis factor (TNF) $\alpha$, sterol regulatory binding protein (SREBP)-1c, fatty acid synthase, stearoyl-carnitine palmitoyl-coenzyme A desaturase, carnitine palmitoylcoenzyme A transferase and inducible nitric oxide synthase (iNOS) were used with $18 \mathrm{~S}$ as an internal control. The probes were obtained from Applied Biosystems (Perkin-Elmer/PE Applied Biosystems, Foster City, CA, USA) and purchased in a ready-for-use form in Assayson-Demand Gene Expression Products. The TaqMan probes were labeled at the $5^{\prime}$ end with the reporter dye fluorescein and at the $3^{\prime}$ end with minor groove binder nonfluorescent quencher. Quantitative PCR was performed in triplicate for each sample on a Step One Plus Real Time System (Applied Biosystems). Each 20- $\mu$ l reaction contained $10 \mu \mathrm{l}$ of TaqMan Fast Universal Master Mix (Applied Biosystems, Darmstadt, Germany), $1 \mu$ l of Gene Expression Mix (Perkin-Elmer/PE Applied Biosystem) and $2 \mu \mathrm{l}$ of cDNA diluted in $7 \mu \mathrm{l}$ RNase-free water. The thermal cycler conditions were $20 \mathrm{~s}$ at $95^{\circ} \mathrm{C}, 40$ cycles of $5 \mathrm{~s}$ at $95^{\circ} \mathrm{C}$ and then $20 \mathrm{~s}$ at $60^{\circ}$. Fold changes in target gene messengerRNA (mRNA) relative to the endogenous $18 \mathrm{~S}$ control were calculated as described in previous studies. ${ }^{25}$

\section{Measurement of TG content}

Hepatic TG content was quantified using a commercial kit (ab65336, Abcam, Cambridge, MA, USA) according to the manufacturer's recommendations. Briefly, lipid extracts were prepared by the homogenization of $50 \mathrm{mg}$ of frozen tissue in a solution of $5 \%$ NP-40. The homogenized tissue was heated to $80-100^{\circ} \mathrm{C}$ in a water bath for 2-5 min, then cooled down to room temperature. The samples were purified and diluted $(1: 10)$ in distilled $\mathrm{H}_{2} \mathrm{O}$ to be measured at $\mathrm{OD}_{570 \mathrm{~mm}}$ according to the manufacturer's instructions.

\section{Statistical analysis}

All results are shown as the mean \pm (s.e.m.). Data were analyzed by nonparametric analysis (Kruskal-Wallis or Mann-Whitney test) or one-way analysis of variance with Tukey's post-hoc analysis. $P<0.05$ was considered statistically significant. All calculations were performed with SPSS version 15.0 software for Windows (SPSS Inc., Chicago, IL, USA). 


\section{RESULTS}

Characteristics of mice fed an HFD with or without experimental materials

The changes in body weight during the 48 weeks of the experiment are shown in Figure 1 and serum biochemical findings are summarized in Table 1. Administering omega-3 did not induce significant changes in body weight independent of UDCA combinations. However, serum TG levels decreased significantly as a result of administering omega-3 alone $(P=0.012)$ or the concomitant administration of omega-3 and UDCA $(P<0.011)$. There was no difference in TG levels between the omega-3 group and omega-3 with UDCA group ( $P=0.995$, when compared with the omega-3 only group). Furthermore, serum alanine aminotransferase decreased when mice were fed omega-3 alone $(P<0.001)$ or in combination with UDCA $(P<0.001)$. There was no difference in alanine aminotransferase levels between the omega-3 group and omega-3 with UDCA group $(P=0.999$, when compared with the omega-3 only group).

Omega-3-attenuated NASH-associated liver fibrosis and adding UDCA further enhanced its anti-fibrotic effect Histological assessment of liver fibrosis was performed using an image analysis system after Sirius Red staining. Administering

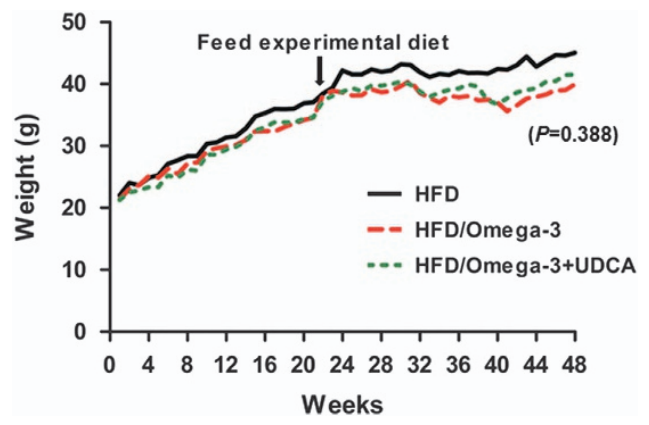

Figure 1 Changes in bodyweight during 48 weeks of the experiment. All the mice were fed a high fat diet (HFD) for 24 weeks and were then divided into three groups. One group $(n=8)$ was fed an HFD only (HFD), another group $(n=8)$ was fed an HFD and administered omega-3 (HFD/Omega-3) and the other group $(n=8)$ was fed an HFD and administered omega-3 in combination with UDCA (HFD/Omega-3+UDCA) for another 24 weeks. omega-3 at a daily dose of $70 \mathrm{mg} \mathrm{kg}^{-1}$ with an HFD-improved histologically evaluated liver fibrosis $(P<0.001$; Figures $2 \mathrm{a}$ and b). Administration of UDCA at a daily dose of $20 \mathrm{mg} \mathrm{kg}^{-1}$ in combination with omega-3 further attenuated HFD-induced liver fibrosis $(P=0.026$, compared with the omega-3 only group; Figure $2 \mathrm{~b})$.

The effect of omega-3 and UDCA on NASH-associated liver fibrosis was also investigated by quantitative analyses of collagen $\alpha 1$ (I) and TGF $\beta$ mRNA expression. Omega-3 alleviated HFDinduced collagen deposition $(P=0.002)$ and administering UDCA in combination with omega-3 further decreased Col $\alpha 1$ (I) expression $(P=0.021$, compared with the omega- 3 only group; Figure $3 \mathrm{a})$. In addition, administering omega- 3 as a supplement to the HFD diminished the mRNA expression of the pro-fibrotic gene TGF $\beta(P=0.012)$, and administering UDCA in combination with omega- 3 further accentuated this effect $(P=0.029$, compared with the omega-3 only group; Figure 3a).

\section{Omega-3 and UDCA-attenuated NASH-associated liver inflammation}

Histological analysis of liver inflammation was carried out after H\&E staining. Administering UDCA in combination with omega-3 ameliorated histologically assessed liver inflammation when compared with that of mice fed an HFD only $(P=0.036$; Figures $2 \mathrm{a}$ and $\mathrm{c})$. In addition, when liver expression of $T N F \alpha$, an inflammatory cytokine, was assessed, administering omega-3 attenuated the TNF $\alpha$ mRNA increase $(P<0.001)$ and adding UDCA further decreased TNF $\alpha$ expression $(P=0.008$, compared with the omega- 3 only group; Figure $3 b$ ).

Omega-3 and UDCA-attenuated hepatic TG accumulation The effect of omega-3 and UDCA on HFD-induced hepatic TG accumulation was evaluated by measuring the tissue TG content. Administering omega-3 with the HFD resulted in decreased hepatic TG content compared with that of mice fed the HFD only $(P=0.029)$. In addition, the combination of UDCA and omega-3 further attenuated hepatic TG accumulation $(P=0.021$; Figure 4$)$.

Table 1 Characterization of mice fed an HFD and administered omega-3 with or without UDCA

\begin{tabular}{|c|c|c|c|}
\hline Diet & $\begin{array}{c}H F D \\
\text { Vehicle }(\mathrm{n}=8)\end{array}$ & $\begin{array}{c}H F D \\
\text { Omega-3 }(\mathrm{n}=8)\end{array}$ & $\begin{array}{c}H F D \\
\text { Omega-3+UDCA }(\mathrm{n}=8)\end{array}$ \\
\hline Serum glucose $\left(\mathrm{mg} \mathrm{dl}^{-1}\right)$ & $116.00 \pm 28.00$ & $120.25 \pm 12.45$ & $146.66 \pm 12.13$ \\
\hline Serum Chol (mg dl-1) & $158.00 \pm 5.19$ & $145.25 \pm 9.07$ & $153.00 \pm 13.40$ \\
\hline Serum ALT $\left(I U I^{-1}\right)$ & $393.00 \pm 26.57$ & $88.25 \pm 11.32^{a}$ & $87.25 \pm 6.67^{a}$ \\
\hline
\end{tabular}

Abbreviations: ALT, alanine aminotransferase; Chol, cholesterol; HFD, high fat diet; Omega-3, omega-3 polyunsaturated fatty acid; TG, triglyceride; UDCA,

ursodeoxycholic acid.

Data are expressed as the mean \pm s.e.

aP $<0.05$, when compared with mice fed HFD alone. 

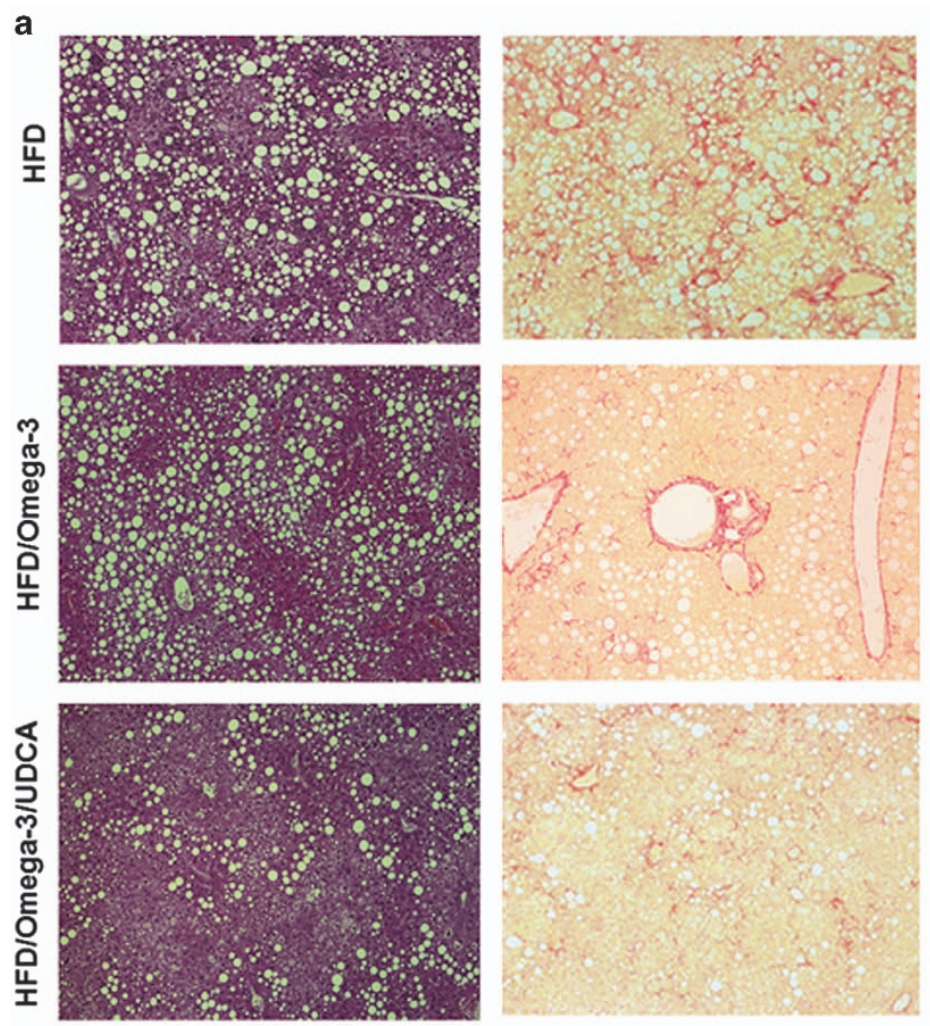

b
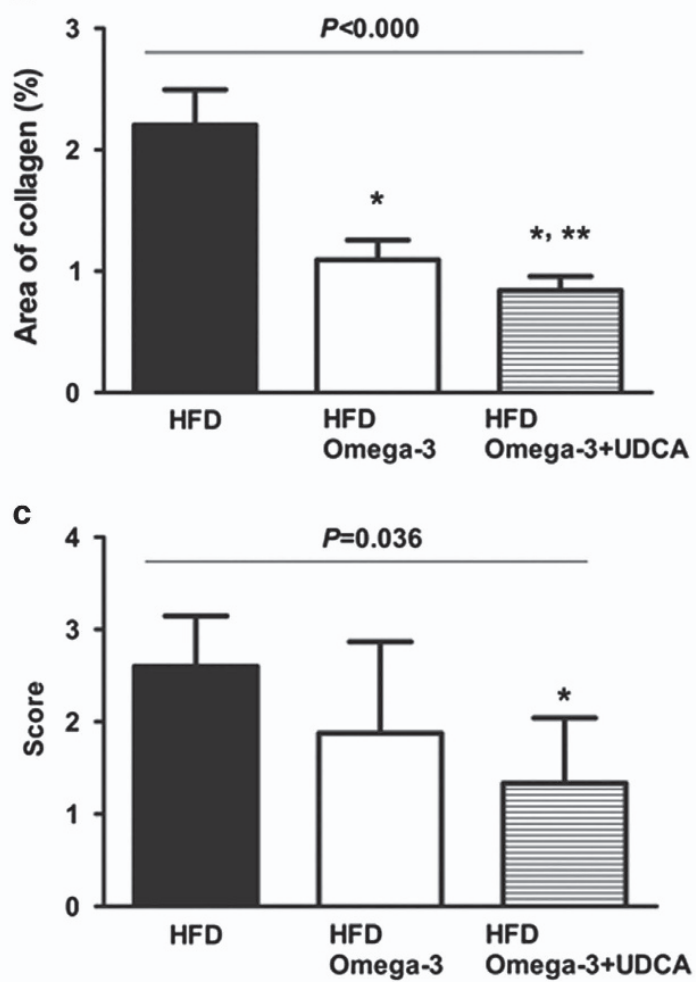

Figure 2 Histological assessment of fibrosis and inflammation in diet-induced NASH. Histological analysis of the liver to evaluate inflammation and fibrosis was performed after H\&E and Sirius Red staining. Mice were fed with a high fat diet (HFD) for 24 weeks and were then divided into three groups. One group $(n=8)$ was fed an HFD only (HFD), another group $(n=8)$ was fed an HFD and administered omega-3 (HFD/Omega-3) and the other group $(n=8)$ was fed an HFD and administered omega-3 in combination with UDCA (HFD/Omega-3+UDCA) for 24 weeks. (a) Representative histological evaluation using H\&E $(\times 100)$ and Sirius Red $(\times 100)$. (b) Percent region of fibrosis. Liver fibrosis was evaluated by calculating the fibrosis ratio using an image analysis system. The total area was the sum of the area of microscopic fields including parenchyma and fibrosis. (c) Inflammation was histologically quantified by counting inflammatory foci in 20 consecutive high-power fields ( $\times 40$ objective). The averaged histological grades are expressed as the following; grade 0 : no foci, grade $1:<2$ foci per high-power field, grade $2: \geqslant 2$ foci per high-power field. ${ }^{*} P<0.05$ when compared with that of the HFD group. ${ }^{* *} P<0.05$ when compared with that of HFD/Omega-3 group.

Omega-3 suppressed the expression of lipogenesis-associated genes and adding UDCA augmented this effect

The mRNA expression of SREBP-1c, which is known to regulate lipogenic enzymes, ${ }^{26}$ was decreased after omega-3 administration in HFD-induced NASH $(P=0.014)$. Administering UDCA in combination with omega-3 further diminished SREBP-1c expression $(P=0.001$, when compared with the omega-3 only group; Figure 3c). The mRNA expression of fatty acid synthase, a gene involved in de novo lipogenesis, decreased when omega-3 was administered $(P=0.035)$, but UDCA did not show an additive effect $(P=0.343$, when compared with the omega-3 only group). The mRNA expression of stearoyl-carnitine palmitoyl-coenzyme A desaturase, another gene involved in de novo lipogenesis, was also reduced when omega- 3 was administered $(P=0.022)$, but there was no significant difference when UDCA was administered in combination with omega-3 $(P=0.083$ when compared with the omega- 3 only group). The mRNA expression of carnitine palmitoyl-coenzyme A transferase, a mitochondrial $\beta$-oxidation enzyme, decreased with omega-3 supplement ingestion $(P=0.045)$, but adding UDCA did not produce a further reduction in carnitine palmitoyl-coenzyme $A$ transferase mRNA expression $(P=0.686$, when compared with the omega-3 only group).

The effect of UDCA on NASH was associated with decreased iNOS mRNA expression

The upregulation of $i N O S$ is known to be associated with liver inflammation and tissue damage, ${ }^{27,28}$ and UDCA has been reported to reduce $i N O S$ in several inflammatory diseases. ${ }^{29,30}$ We evaluated whether the beneficial effect of UDCA on NASH in our study was associated with decreased iNOS mRNA expression. Administration of omega-3 with the HFD resulted in diminished iNOS mRNA $(P=0.012)$, and adding UDCA in combination with omega-3 further suppressed iNOS mRNA expression ( $P=0.029$, when compared with the omega- 3 only group; Figure 3d).

\section{DISCUSSION}

Although several previous studies have reported that omega-3 attenuates diet-induced $\mathrm{NASH},{ }^{31-33}$ to our knowledge, this 
a

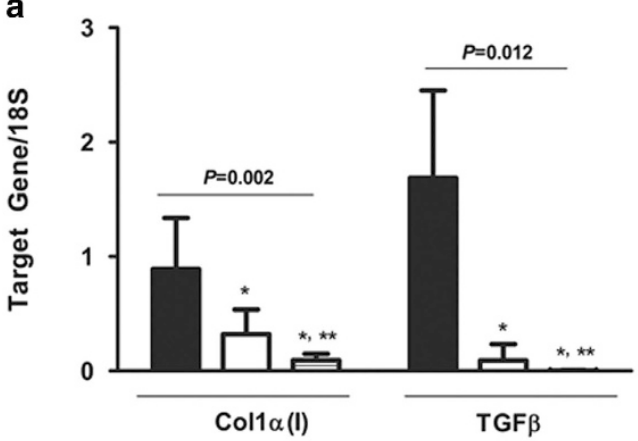

C

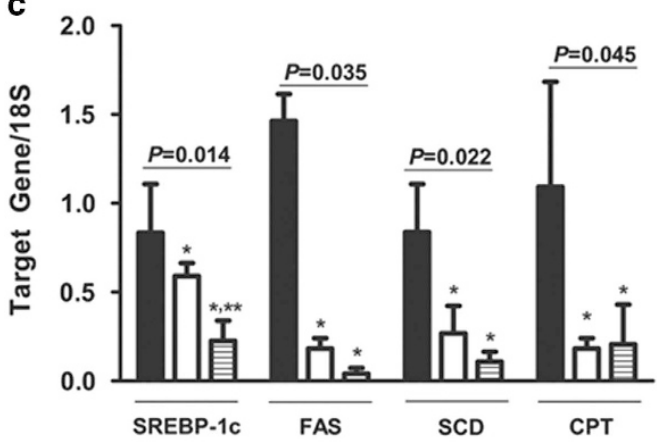

$\square$ HFD

HFD/Omega-3

\# HFD/Omega-3+UDCA

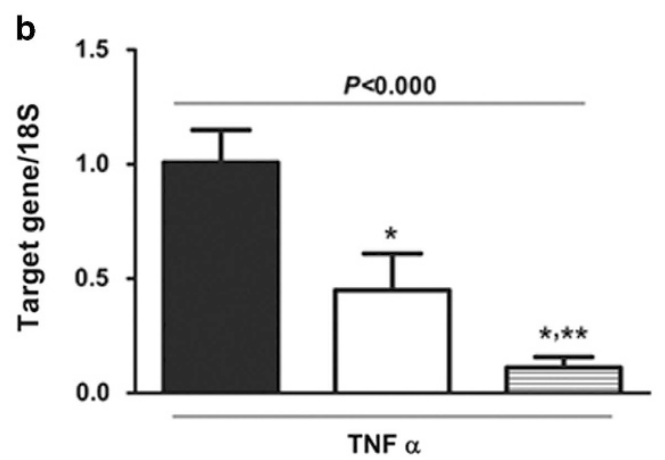

d

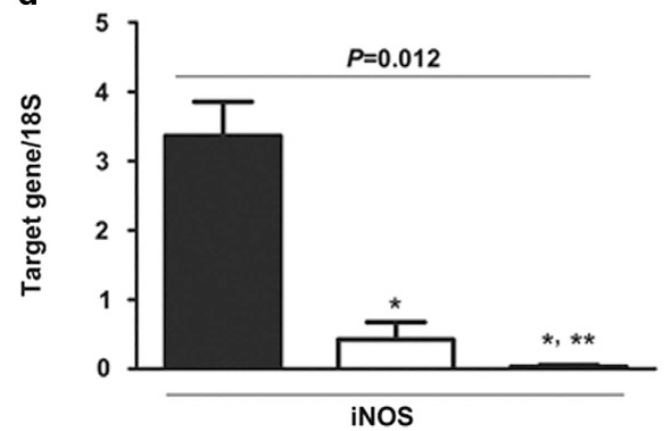

Figure 3 Changes in gene expression related to fibrosis, inflammation, steatosis and oxidative stress. Mice were fed a high fat diet (HFD) for 24 weeks and were then divided into three groups. One group $(n=8)$ was fed an HFD only (HFD), another group ( $n=8)$ was fed an HFD and administered omega-3 (HFD/Omega-3) and the other group $(n=8)$ was fed an HFD and administered omega-3 in combination with UDCA (HFD/Omega-3+UDCA) for 24 weeks. (a) Expression of fibrosis related genes in the liver. Quantitative analysis of liver Col $\alpha(I)$ and TGF $\beta$ mRNA, markers of fibrosis, decreased when omega-3 was added to HFD. The HFD/Omega-3+UDCA group exhibited decreased Colo $1(I)$ and TGF $\beta$ expression when compared with that of the HFD/Omega-3 group. (b) Expression of inflammation-related $T N F \alpha$ in the liver. Quantitative analysis of liver TNF $\alpha$ mRNA was decreased in the HFD/Omega-3 and HFD/Omega-3+UDCA groups when compared with the HFD group. The HFD/Omega-3+UDCA group had decreased TNF $\alpha$ expression when compared with that of the HFD/Omega-3 group. (c) Expression of lipogenic genes. mRNA expression of SREBP-1c, the lipogenic gene regulator, was decreased in the HFD/Omega-3 and HFD/Omega-3+UDCA groups when compared with the HFD group. The HFD/Omega-3+UDCA group had decreased SREBP-1C expression when compared with that of the HFD/Omega-3 group. mRNA expression of fatty acid synthase and stearoyl-carnitine palmitoyl-coenzyme A desaturase, genes involved in de novo lipogenesis, were significantly decreased when omega-3 was administered. However, no additive effect was found with UDCA. CPA, a fatty acid oxidation related gene, was diminished by administering omega-3 with an HFD, but no further reduction was noticed by administering UDCA in combination with omega-3. (d) Expression of an oxidative stress-related gene. Decreased liver iNOS mRNA expression was observed with omega-3 as a supplement to HFD and adding UDCA further augmented this effect. ${ }^{*} P<0.05$ when compared with that of the HFD group. ${ }^{* *} P<0.05$ when compared with that of HFD/Omega-3 group.

is the first study to report an additive effect of omega-3 and UDCA on NASH. In accordance with other studies, our study also demonstrated that omega-3 alone could alleviate liver fibrosis identified by decreased collagen $\alpha 1(I)$ mRNA expression. However, administering UDCA in combination with omega-3 further reduced fibrosis at the mRNA level and also further attenuated histologically assessed liver fibrosis when compared with the group to which omega-3 was administered alone. It has been repeatedly suggested that liver fibrosis is one of the most important factors in forecasting the progression of $\mathrm{NASH},{ }^{34-36}$ and an additive effect of omega-3 and UDCA in diminishing NASH-associated fibrosis makes this combination a possible candidate for the treatment of NASH.

TGF $\beta$ has been identified as a key regulator of liver fibrosis that exerts its profibrogenic effect through hepatic stellate cells. ${ }^{37}$ The role of TGF $\beta$ signaling in the progression of NASH-associated fibrosis has also been reported in several studies, making it an attractive target for treatment. ${ }^{38,39}$ Our study demonstrated that omega-3 and UDCA had an additive effect in suppressing TGF $\beta$ mRNA expression, although TGF $\beta$ is not suggested as a target for this combination therapy, according to our results. 


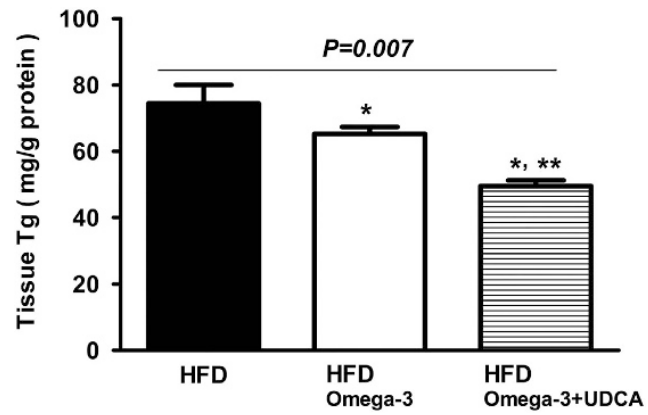

Figure 4 Hepatic triglyceride content. Hepatic triglyceride content was measured in frozen liver tissue using a commercial kit. The mice were fed a high fat diet (HFD) for 24 weeks and were then divided into three groups. One group $(n=8)$ was fed an HFD only (HFD), another group $(n=8)$ was fed an HFD and administered omega-3 (HFD/Omega-3) and the other group $(n=8)$ was fed an HFD and administered omega-3 in combination with UDCA (HFD/ Omega-3+UDCA) for another 24 weeks. ${ }^{*} P<0.05$ when compared with that of the HFD group. ${ }^{*} P<0.05$ when compared with that of the HFD/Omega-3 group.

Even with the promising results of either omega-3 or UDCA on experimentally induced NASH, neither omega-3 nor UDCA has succeeded in showing a significant therapeutic effect when tested in NASH patients. Randomized controlled clinical trials investigating the effect of either omega-3 or UDCA on NASH in patients have failed to show significant improvement when compared with a placebo. ${ }^{17,18}$ However, a systemic review has suggested that negative results on the beneficial role of these agents might be attributed to improperly constructed study designs including suboptimal drug dosage and study duration. ${ }^{19}$ Meanwhile, several studies testing UDCA-based combination regimens, such as UDCA with vitamin E, resulted in biochemical or histological improvements in NASH patients. ${ }^{40,41}$ However, as described earlier, there has been a report showing the adverse effects of long-term vitamin $\mathrm{E}$ consumption, ${ }^{20}$ and safety should be the utmost concern in medical treatments of NASH. No serious side-effects have been recorded with UDCA treatment, and a human study using $25 \mathrm{mg} \mathrm{kg}^{-1}$ of UDCA, which was higher than the dose used in our study, has been carried out without reporting any serious adverse events. ${ }^{42}$ As for omega- 3 , the currently recommended maximal dose is $4000 \mathrm{mg}$ per day, which is equivalent to the dose used in this study.

In addition to liver fibrosis, inflammation is also known to have a major role in the development and progression of NASH. ${ }^{23,43}$ Although administering omega-3 alone failed to show significant histologic changes, administering UDCA in combination with omega-3 resulted in histologically improved inflammation in diet-induced NASH, suggesting an additive effect. A previous study also demonstrated decreased expression of the proinflammatory cytokine TNF $\alpha$ after omega-3 supplementation, ${ }^{44}$ which is in accordance with our study results.

TG accumulation in the liver is known to be controlled by SREBP-1c, a transcriptional factor that regulates lipogenic enzymes. ${ }^{45,46}$ Several studies have reported that omega-3 supplementation downregulated SREBP-1c gene expression, ${ }^{6-9}$ and a recent study demonstrated that UDCA mediated lipogenesis by repressing SREBP-1c expression. ${ }^{47}$ Our study suggested an additive effect of omega-3 and UDCA for suppressing SREBP-1c, although this study could not reveal an additive effect of UDCA for regulating genes related to de novo lipogenesis or fatty acid oxidation.

Although our study does not delineate the mechanistic role of omega-3 and UDCA-combined treatment for improving $\mathrm{NASH}$, suppressed $i N O S$ expression suggests that reduced liver damage from oxidative stress may be a possible explanation. Increased iNOS production has been demonstrated in fructoseinduced hepatic steatosis, ${ }^{48}$ and suppressed iNOS production has been associated with the use of anti-inflammatory drugs. ${ }^{49}$ Studies investigating more detailed mechanisms are needed in the future.

In conclusion, our study demonstrated an additive effect of omega-3 and UDCA for alleviating fibrosis and inflammation in diet-induced NASH. Our study also demonstrated the additive effect of omega-3 and UDCA for suppressing the lipogenic gene $S R E B P-1 c$ and the oxidative stress related gene iNOS. These results suggest that the combination of omega-3 and UDCA may be a possible treatment for NASH, although validation using different NASH models and human studies are necessary.

\section{CONFLICT OF INTEREST}

The authors declare no conflict of interest.

\section{ACKNOWLEDGEMENTS}

This study was supported by a faculty research grant from Yonsei University College of Medicine (6-2009-0098).

1 Angulo P. Gl Epidemiology: nonalcoholic fatty liver disease. Aliment Pharmacol Ther 2007; 25: 883-889.

2 Argo CK, Caldwell SH. Epidemiology and Natural History of Non-Alcoholic Steatohepatitis. Clin Liver Dis 2009; 13: 511-531.

3 Angulo P. Long-term mortality in nonalcoholic fatty liver disease: is liver histology of any prognostic significance? Hepatology 2010; 51: 373-375.

4 Bhala N, Angulo P, van der Poorten D, Lee E, Hui JM, Saracco G et al. The natural history of nonalcoholic fatty liver disease with advanced fibrosis or cirrhosis: an International Collaborative Study. Hepatology 2011; 54: $1208-1216$.

5 Yokoyama M, Origasa H, Matsuzaki M, Matsuzawa Y, Saito Y, Ishikawa Y et al. Effects of eicosapentaenoic acid on major coronary events in hypercholesterolaemic patients (JELIS): a randomised openlabel, blinded endpoint analysis. Lancet 2007; 369: 1090-1098.

$6 \mathrm{Kim}$ HJ, Takahashi M, Ezaki O. Fish oil feeding decreases mature sterol regulatory element-binding protein 1 (SREBP-1) by down-regulation of SREBP-1c mRNA in mouse liver. A possible mechanism for downregulation of lipogenic enzyme mRNAs. J Biol Chem 1999; 274: 25892-25898.

7 Yahagi N, Shimano H, Hasty AH, Amemiya-Kudo M, Okazaki H, Tamura Y et al. A crucial role of sterol regulatory element-binding protein-1 in the regulation of lipogenic gene expression by polyunsaturated fatty acids. J Biol Chem 1999; 274: 35840-35844.

$8 \mathrm{Xu} \mathrm{J}$, Nakamura MT, Cho HP, Clarke SD. Sterol regulatory element binding protein-1 expression is suppressed by dietary polyunsaturated fatty acids. A mechanism for the coordinate suppression of lipogenic genes by polyunsaturated fats. J Biol Chem 1999; 274: 23577-23583. 
9 Botolin D, Wang Y, Christian B, Jump DB. Docosahexaneoic acid (22:6, $n-3)$ regulates rat hepatocyte SREBP-1 nuclear abundance by Erk- and $26 \mathrm{~S}$ proteasome-dependent pathways. J Lipid Res 2006; 47: 181-192.

10 Zhu FS, Liu S, Chen XM, Huang ZG, Zhang DW. Effects of n-3 polyunsaturated fatty acids from seal oils on nonalcoholic fatty liver disease associated with hyperlipidemia. World J Gastroenterol 2008; 14 : $6395-6400$

11 Spadaro L, Magliocco O, Spampinato D, Piro S, Oliveri C, Alagona C et al. Effects of $n-3$ polyunsaturated fatty acids in subjects with nonalcoholic fatty liver disease. Dig Liver Dis 2008; 40: 194-199.

12 Parker HM, Johnson NA, Burdon CA, Cohn JS, O'Connor HT, George J. Omega-3 supplementation and non-alcoholic fatty liver disease: a systematic review and meta-analysis. J Hepatol 2012; 56: 944-951.

13 Chamulitrat W, Burhenne J, Rehlen T, Pathil A, Stremmel W. Bile saltphospholipid conjugate ursodeoxycholyl lysophosphatidylethanolamide as a hepatoprotective agent. Hepatology 2009; 50: 143-154.

14 Pathil A, Warth A, Chamulitrat W, Stremmel W. The synthetic bile acidphospholipid conjugate ursodeoxycholyl lysophosphatidylethanolamide suppresses TNFalpha-induced liver injury. J Hepatol 2011; 54: 674-684.

15 Mitsuyoshi H, Nakashima T, Sumida Y, Yoh T, Nakajima Y, Ishikawa H et al. Ursodeoxycholic acid protects hepatocytes against oxidative injury via induction of antioxidants. Biochem Biophys Res Commun 1999; 263 : 537-542.

16 Uzun MA, Koksal N, Aktas S, Gunerhan Y, Kadioglu H, Dursun N et al. The effect of ursodeoxycholic acid on liver regeneration after partial hepatectomy in rats with non-alcoholic fatty liver disease. Hepatol Res 2009; 39: 814-821.

17 Leuschner UFH, Lindenthal B, Herrmann G, Arnold JC, Rossle M, Cordes $\mathrm{HJ}$ et al. High-dose ursodeoxycholic acid therapy for nonalcoholic steatohepatitis: a double-blind, randomized, placebo-controlled trial. Hepatology 2010; 52: 472-479.

18 Dasarathy S, Dasarathy J, Khiyami A, Yerian LM, Sargent R, Hawkins CA et al. Randomized controlled trial of omega 3 fatty acids in the treatment of non alcoholic steatohepatitis in type 2 diabetes mellitus. Hepatology 2013; 58: 518a-518a.

19 Xiang Z, Chen YP, Ma KF, Ye YF, Zheng L, Yang YD et al. The role of ursodeoxycholic acid in non-alcoholic steatohepatitis: a systematic review. BMC Gastroenterol 2013; 13: 140.

20 Miller ER, Pastor-Barriuso R, Dalal D, Riemersma RA, Appel LJ, Guallar E. Meta-analysis: high-dosage vitamin $\mathrm{E}$ supplementation may increase allcause mortality. Ann Intern Med 2005; 142: 37-46.

21 Brunt EM. Nonalcoholic steatohepatitis: definition and pathology. Semin Liver Dis 2001; 21: 3-16.

22 Kirsch R, Clarkson V, Shephard EG, Marais DA, Jaffer MA, Woodburne VE et al. Rodent nutritional model of non-alcoholic steatohepatitis: species, strain and sex difference studies. J Gastroenterol Hepatol 2003; 18: 1272-1282.

23 Kleiner DE, Brunt EM, Van Natta M, Behling C, Contos MJ, Cummings OW et al. Design and validation of a histological scoring system for nonalcoholic fatty liver disease. Hepatology 2005; 41: 1313-1321.

24 O'Brien MJ, Keating NM, Elderiny S, Cerda S, Keaveny AP, Afdhal NH et al. An assessment of digital image analysis to measure fibrosis in liver biopsy specimens of patients with chronic hepatitis C. Am J Clin Pathol 2000; 114: 712-718.

25 Livak KJ, Schmittgen TD. Analysis of relative gene expression data using real-time quantitative PCR and the 2(T)(-Delta Delta C) method. Methods 2001; 25: 402-408.

26 Nagaya T, Tanaka N, Suzuki T, Sano K, Horiuchi A, Komatsu M et al. Down-regulation of SREBP-1C is associated with the development of burned-out NASH. J Hepatol 2010; 53: 724-731.

27 Droge W. Free radicals in the physiological control of cell function. Physiol Rev 2002; 82: 47-95.

28 Brenner C, Galluzzi L, Kepp 0, Kroemer G. Decoding cell death signals in liver inflammation. J Hepatol 2013; 59: 583-594.

29 Carotti S, Guarino MP, Cicala M, Perrone G, Alloni R, Segreto F et al. Effect of ursodeoxycholic acid on inflammatory infiltrate in gallbladder muscle of cholesterol gallstone patients. Neurogastroenterol Motil 2010; 22: e232.

30 Moon SJ, Jeong JH, Jhun JY, Yang EJ, Min JK, Choi JY et al. Ursodeoxycholic acid ameliorates pain severity and cartilage degeneration in monosodium iodoacetate-induced osteoarthritis in rats. Immune Netw 2014; 14: 45-53.

31 Ishii H, Horie Y, Ohshima S, Anezaki Y, Kinoshita N, Dohmen T et al. Eicosapentaenoic acid ameliorates steatollepatitis and hepatocellular carcinoma in hepatocyte-specific Pten-deficient mice. J Hepatol 2009; 50: 562-571.

32 Kajikawa S, Imada K, Takeuchi T, Shimizu Y, Kawashima A, Harada T et al. Eicosapentaenoic acid attenuates progression of hepatic fibrosis with inhibition of reactive oxygen species production in rats fed methionineand choline-deficient diet. Dig Dis Sci 2011; 56: 1065-1074.

33 Depner CM, Philbrick KA, Jump DB. Docosahexaenoic acid attenuates hepatic inflammation, oxidative stress, and fibrosis without decreasing hepatosteatosis in a Ldlr(-/-) mouse model of western diet-induced nonalcoholic steatohepatitis. J Nutr 2013; 143: 315-323.

34 Harrison SA, Torgerson S, Hayashi PH. The natural history of nonalcoholic fatty liver disease: a clinical histopathological study. Am J Gastroenterol 2003; 98: 2042-2047.

35 Fassio E, Alvarez E, Dominguez N, Landeira G, Longo C. Natural history of nonalcoholic steatohepatitis: a longitudinal study of repeat liver biopsies. Hepatology 2004; 40: 820-826.

36 Adams LA, Sanderson S, Lindor KD, Angulo P. The histological course of nonalcoholic fatty liver disease: a longitudinal study of 103 patients with sequential liver biopsies. J Hepatol 2005; 42: 132-138.

37 Friedman SL. Mechanisms of hepatic fibrogenesis. Gastroenterology 2008; 134: 1655-1669.

38 Cayon A, Crespo J, Mayorga M, Guerra A, Pons-Romero F. Increased expression of $\mathrm{Ob}-\mathrm{Rb}$ and its relationship with the overexpression of TGFbeta 1 and the stage of fibrosis in patients with nonalcoholic steatohepatitis. Liver Int 2006; 26: 1065-1071.

39 Klein S, Mittendorfer B, Eagon JC, Patterson B, Grant L, Feirt N et al. Gastric bypass surgery improves metabolic and hepatic abnormalities associated with nonalcoholic fatty liver disease. Gastroenterology 2006; 130: 1564-1572.

40 Dufour JF, Oneta CM, Gonvers JJ, Bihl F, Cerny A, Cereda JM et al. Randomized placebo-controlled trial of ursodeoxycholic acid with vitamin $\mathrm{E}$ in nonalcoholic steatohepatitis. Clin Gastroenterol Hepatol 2006; 4: 1537-1543.

41 Pietu F, Guillaud O, Walter T, Vallin M, Hervieu V, Scoazec JY et al. Ursodeoxycholic acid with vitamin $\mathrm{E}$ in patients with nonalcoholic steatohepatitis: long-term results. Clin Res Hepatol Gastroenterol 2012; 36: 146-155.

42 Azzaroli F, Raspanti ME, Simoni P, Montagnani M, Lisotti A, Cecinato P et al. High doses of ursodeoxycholic acid up-regulate the expression of placental breast cancer resistance protein in patients affected by intrahepatic cholestasis of pregnancy. PLOS ONE 2013; 8: e64101.

43 Tilg $\mathrm{H}$, Moschen AR. Evolution of inflammation in nonalcoholic fatty liver disease: the multiple parallel hits hypothesis. Hepatology 2010; 52: 1836-1846.

44 Stienstra R, Mandard S, Patsouris D, Maass C, Kersten S, Muller M. Peroxisome proliferator-activated receptor alpha protects against obesityinduced hepatic inflammation. Endocrinology 2007; 148: 2753-2763.

45 Browning JD, Horton JD. Molecular mediators of hepatic steatosis and liver injury. J Clin Invest 2004; 114: 147-152.

46 Ferre P, Foufelle F. SREBP-1c transcription factor and lipid homeostasis: clinical perspective. Horm Res 2007; 68: 72-82.

47 Lee JM, Gang GT, Kim DK, Kim YD, Koo SH, Lee CH et al. Ursodeoxycholic acid inhibits liver $X$ receptor alpha-mediated hepatic lipogenesis via induction of the nuclear corepressor SMILE. J Biol Chem 2014; 289: 1079-1091.

48 Spruss A, Kanuri G, Wagnerberger S, Haub S, Bischoff SC, Bergheim I. Toll-Like receptor 4 is involved in the development of fructose-induced hepatic steatosis in mice. Hepatology 2009; 50: 1094-1104.

49 Moeslinger T, Friedl R, Spieckermann PG. Inhibition of inducible nitric oxide synthesis by azathioprine in a macrophage cell line. Life Sci 2006; 79: 374-381.

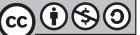

This work is licensed under a Creative Commons Attribution-NonCommercial-ShareAlike 3.0 Unported License. The images or other third party material in this article are included in the article's Creative Commons license, unless indicated otherwise in the credit line; if the material is not included under the Creative Commons license, users will need to obtain permission from the license holder to reproduce the material. To view a copy of this license, visit http:// creativecommons.org/licenses/by-nc-sa/3.0/ 